\title{
Poor Sleep Quality is an Independent Risk Factor for Acquired Premature Ejaculation
}

\author{
Wei Zhang $\mathbb{D}^{\prime}{ }^{\prime *}$, Yuyang Zhang',*, Jingjing Gao', Dangwei Peng', Yao Zhang', Xu Wu', Guodong Liu', \\ Yutian Dai ${ }^{2}$, Hui Jiang ${ }^{3}$, Xiansheng Zhang' \\ 'Department of Urology, The First Affiliated Hospital of Anhui Medical University, Hefei, Anhui, People's Republic of China; ${ }^{2}$ Department of Urology, \\ Gulou Hospital of Nanjing University, Nanjing, Jiangsu Province, People's Republic of China; ${ }^{3}$ Department of Reproductive Medicine Center, Peking \\ University Third Hospital, Beijing, People's Republic of China \\ *These authors contributed equally to this work \\ Correspondence: Xiansheng Zhang; Hui Jiang, Email xiansheng-zhang@I63.com; jianghui55@l63.com
}

Purpose: To determine the role of poor sleep quality as a risk factor for acquired premature ejaculation (APE) after considering the various risk factors, such as ages, lower urinary tract symptoms (LUTS), anxiety, depression, and erectile dysfunction.

Methods: This study presents a multivariate analysis to identify risk factors for PE, including the covariate of age, International Prostate Symptom Score (IPSS), General Anxiety Disorder-7 (GAD-7) score, Patient Health Questionnaire-9 (PHQ-9) score, International Index of Erectile Function (IIEF) score, and Pittsburgh Sleep Quality Index (PSQI). Acquired PE was defined as self-reported intravaginal ejaculation latency time $\leq 3$ minutes, and poor sleep quality was diagnosed using the Pittsburgh Sleep Quality Index tool.

Results: A total of 349 men were enrolled in the study after completing the questionnaires and the medical history survey. Among 349 men, 203 individuals (58.17\%) suffered from acquired PE. The IIEF-5 score, IPSS, GAD-7 score, PHQ-9 score, and PSQI score of the population with PE were significantly different from the non-PE group. Further multivariate analysis showed that erectile dysfunction, depression, severe prostatitis-like symptoms, and poor sleep quality were high-risk factors of APE. Additionally, our study showed that premature ejaculation diagnostic tool (PEDT) score was associated with IPSS/GAD-7/PHQ-9/PSQI scores positively and associated with IIEF-5 scores negatively. The stratified analysis of sleep quality showed that APE patients with different sleep qualities have different prevalence rates of anxiety, depression, prostatitis-like symptoms, and erectile function.

Conclusion: In general, sleep quality may be a potential risk factor for patients with acquired premature ejaculation. Our research revealed the impact of sleep quality on premature ejaculation and provided new viewpoints for further understanding and perfecting the pathogenesis of premature ejaculation.

Keywords: sleep quality, acquired premature ejaculation, multivariate analysis

\section{Introduction}

Premature ejaculation (PE) is one of the most common sexual dysfunctions, present in about $14.0 \%$ to $19.5 \%$ of all adult men. ${ }^{1,2}$ Schapiro $^{3}$ was the first to describe two types of PE: primary PE (PPE, or lifelong PE) and secondary PE (SPE, or acquired PE). In 2014, the International Society for Sexual Medicine (ISSM) defined Lifelong PE and acquired PE in detail. ${ }^{4}$ Recently, Colonnello et $\mathrm{al}^{5}$ redefines a sexual medicine paradigm: subclinical premature ejaculation as a new taxonomic entity included subclinical premature ejaculation as a new classification entity, which includes patients who do not completely fulfil the definition criteria for PE, and frequently with other sexual comorbidities, such as erectile dysfunction (ED). The etiology of PE has been traditionally divided into psychogenic and biogenic factors. Furthermore, PE is likely a symptom with a multifactorial etiology with coexisting genetics, neurobiological, endocrine, urologic, intrapsychic, and relational factors. ${ }^{6}$

Sleep that is insufficient, misaligned, or disrupted causes hypersomnolence and neuropsychological deficits, ${ }^{7}$ adversely affect cardiometabolic health and is increasingly recognized to impair other biological processes that lead to conditions important to men, such as hypogonadism, erectile dysfunction, and infertility. ${ }^{8-10}$ 
However, few studies have focused on the relationship between sleep and male ejaculation. Serotonin and its receptors in the central system were confirmed to play an essential role in central ejaculation control. ${ }^{11} 5$-HT in the central nervous system can inhibit the ejaculation reflex, and when its function is disturbed, such as 5-HT receptor dysfunction, the ejaculation latency will be shortened, resulting in premature ejaculation. ${ }^{12}$ At the same time, studies have found that 5-HT in the central system also plays a vital role in regulating the body's sleep-wake and emotional state. ${ }^{13}$ Animal experiments have shown that sleep deprivation in rats will affect the expression of central 5-HT receptors, and causes the sensitivity of the receptor to change. ${ }^{14}$ In addition, studies have confirmed that sleep deprivation interferes with the function of the reproductive system and sexual behavior of male rats, including the ejaculation function. ${ }^{15}$ Consequently, we speculate that sleep may be closely related to the central ejaculation control, and sleep disorder may be one of the risk factors for premature ejaculation. Furthermore, this study presents a multivariate analysis factoring in various comorbidities as well as the effects of age, lower urinary tract symptoms (LUTS), anxiety, depression, and erectile dysfunction, which could influence the presentation of PE.

\section{Materials and Methods}

\section{Study Population}

Patients with primary premature ejaculation have a specific genetic susceptibility to rapid ejaculation from their first sexual life. Compared with patients with primary premature ejaculation, acquired premature ejaculation is usually accompanied by comorbidities such as prostatitis and erectile dysfunction, so we excluded patients with primary premature ejaculation.

From October 2020 to June 2021, a total of 320 patients who complained of "rapid ejaculation" were evaluated in the Andrology Clinic of the First Affiliated Hospital of Anhui Medical University. We excluded 93 patients with primary premature ejaculation and 24 patients who failed to meet the inclusion criteria. Finally, 203 patients with acquired premature ejaculation were included in the study.

The inclusion criteria are as follows: (a) Over 18 years old; (b) The patient maintained a stable sexual relationship with a heterosexual partner for at least six months. (c) The first time turns to andrologists for their confusion; (d) Does not suffer from other neurological diseases or other organic diseases that affect sleep and ejaculation function; (e) Not taking drugs that may affect sexual function and sleep; The control group was composed of 146 healthy adult male patients who underwent physical examination in the outpatient department, without any diseases or drugs history that could affect sleep and ejaculation function.

This study complied with all principles of the Declaration of Helsinki. The Ethics Committee of the First Affiliated Hospital of Anhui Medical University has approved the study. Written informed consent was obtained from each participant before data collection.

\section{Data Collection}

The investigation collected research data mainly through paper questionnaires. After the patients completed the questionnaire, experienced andrologists examined these items to ensure stable and reliable results. The paper questionnaire includes the following items: (a) demographic information, such as age, height, weight, marital status, etc.; (b) detailed sexual and related medical history; (c) self-estimated IELT; (d) the scale of International Index of Erectile Function-5 (IIEF-5); ${ }^{16}$ (e) premature ejaculation diagnostic tool (PEDT) ${ }^{17}$ (f) International Prostate Symptom Score (IPSS); ${ }^{18}$ (g) anxiety and depression, measured with General Anxiety Disorder-7 (GAD-7) ${ }^{19}$ and Patient Health Questionnaire-9 (PHQ-9) ${ }^{20}$ and (h) Pittsburgh sleep quality index (PSQI). ${ }^{21}$

\section{Data Analysis}

Data analysis was conducted using IBM SPSS Statistics for Windows, version 24 (SPSS Inc, Chicago, IL, USA). Kolmogorov-Smirnov test was used to test the normality of data samples. The Student test or Mann-Whitney test was used to compare the differences between groups according to whether the continuous variables conform to the normal distribution. The classified data between groups were analyzed and compared by Pearson chi-square test or Fisher exact 
Table I Demographics Characteristics of Men with and without Acquired Premature Ejaculation

\begin{tabular}{|c|c|c|c|c|}
\hline Demographic Characteristics & All $(n=349)$ & Non-PE $(n=146)$ & Acquired PE (n=203) & $\mathbf{P}$ \\
\hline Age (yr.) & $30.27 \pm 5.47$ & $29.25 \pm 6.64$ & $31.00 \pm 4.32$ & 0.03 \\
\hline$\leq 30$ & 166 & 78 (53.42\%) & $88(43.35 \%)$ & \\
\hline$>30$ & 183 & $68(46.58 \%)$ & II 5 (56.65\%) & \\
\hline BMI $\left(\mathrm{kg} / \mathrm{m}^{2}\right)$ & $24.73 \pm 4.59$ & $23.76 \pm 3.92$ & $25.42 \pm 4.91$ & 0.002 \\
\hline$<18.5$ & 29 & $12(8.22 \%)$ & 17 (8.37\%) & \\
\hline $18.5-24$ & 118 & 59 (40.4l\%) & 59 (29.06\%) & \\
\hline$>24$ & 202 & 75 (5I.37\%) & 127 (62.56\%) & \\
\hline \multicolumn{5}{|l|}{ Personal history } \\
\hline Smoking & 92 & 29 (19.86\%) & $63(31.03 \%)$ & 0.019 \\
\hline Alcohol use & 70 & 29 (19.86\%) & $4 \mid(20.20 \%)$ & 0.939 \\
\hline Regular exercise & 40 & 21 (14.38\%) & $19(9.36 \%)$ & 0.146 \\
\hline \multicolumn{5}{|l|}{ Comorbid conditions } \\
\hline HTN & 52 & $21(14.38 \%)$ & $31(15.27 \%)$ & 0.818 \\
\hline DM & 28 & $13(8.90 \%)$ & 15 (7.39\%) & 0.607 \\
\hline
\end{tabular}

Notes: Values are presented as mean \pm standard deviation or number (\%). Regular exercise: twice a week, at least 30 minutes each time. Abbreviations: PE, premature ejaculation; BMI, body mass index; HTN, hypertension; DM, diabetes mellitus.

test, and the correlation was studied by Pearson correlation analysis or Spearman rank correlation analysis. Binary Logistic regression model was used to evaluate the risk factors associated with acquired premature ejaculation. Continuous variables were expressed as averages with standard deviations (average \pm SD). Numbers with percentages represented classification variables. Statistical significance was defined as $\mathrm{p}<0.05$.

\section{Results}

\section{Patient Characteristics}

A total of 349 men were included in the study after completing the questionnaires and medical history investigation. Among 349 men, 203 subjects (58.17\%) had acquired PE (IELT $\leq 3$ min). The mean ages of the acquired PE and control groups were $31.00 \pm 4.32$ years and $29.25 \pm 6.64$ years, respectively, with significant differences between the groups $(\mathrm{P}<0.05)$. Table 1 shows more demographic characteristics for participants.

\section{Outcomes of the IELT, PEDT, IIEF-5, IPSS, GAD-7, PHQ-9 and PSQI}

The prevalence of ED, anxiety, depression and poor sleep quality in the PE group was 52.70\% (102/203), 73.40\% (149/ 203), 83.74\% (170/203), and 75.37\% (153/203), respectively. In the non-PE group, 10 (6.85\%) men reported ED, 41 $(28.08 \%)$ reported anxiety, $86(58.90 \%)$ reported depression, and $70(47.96 \%)$ men reported poor sleep quality. The PEDT score, IIEF-5 score, IPSS, GAD-7 score, PHQ-9 score, and SQI score of the population with PE were significantly different from the non-PE group ( $p<0.001$ for all, see Table 2 and Figure 1).

\section{Study on the Correlation Between PEDT and the Clinical Characteristics of Patients}

Correlation analysis was conducted to discover whether the PEDT score was correlated with the clinical characteristics of APE patients. After testing the data, Pearson correlation analysis was performed on the data following normal distribution, and the variables of non-normal distribution were analyzed by correlation analysis, which included: IIEF-5 score, IPSS, GAD-7 score, PHQ-9 score, and PSQI. The results showed that the PEDT score in patients with APE was negatively correlated with IIEF-5 score $(r=-0.364, p<0.001$; Figure $2 A)$, and positively correlated with IPSS $(r=0.284$, $p<0.001$; Figure 2B), GAD-7 score $(r=0.230, p<0.001$; Figure 2C), PHQ-9 score $(r=0.196, p=0.008$; Figure 2D) and PSQI $(r=0.310, p<0.001$; Figure 2E). 
Table 2 IELT, PEDT, IIEF-5, IPSS, GAD-7, PHQ-9 and PSQI for Men with and without Acquired Premature Ejaculation

\begin{tabular}{|c|c|c|c|}
\hline Characteristic & Non-PE $(n=146)$ & Acquired PE $(n=203)$ & $\mathbf{P}$ \\
\hline \multicolumn{4}{|l|}{ IELT, min } \\
\hline$<1$ & 0 & $10(4.93 \%)$ & \\
\hline $1-2$ & 14 (9.59\%) & 71 (34.98\%) & \\
\hline$>2$ & $132(90.41 \%)$ & $122(60.10 \%)$ & \\
\hline PEDT score & $5.64 \pm 3.31$ & $14.90 \pm 3.02$ & $<0.001$ \\
\hline IIEF-5 score & $23.33 \pm 1.63$ & $17.16 \pm 6.57$ & $<0.001$ \\
\hline $5-7$ & $2(1.37 \%)$ & $14(6.90 \%)$ & \\
\hline $8-11$ & $2(1.37 \%)$ & $36(17.73 \%)$ & \\
\hline$|2-2|$ & $6(4.11 \%)$ & 57 (28.08\%) & \\
\hline $22-25$ & 136 (93.15\%) & $96(47.29 \%)$ & \\
\hline IPSS score & $1.46 \pm 2.99$ & $8.91 \pm 7.97$ & $<0.001$ \\
\hline $0-7$ & I 44 (98.63\%) & $123(60.59 \%)$ & \\
\hline $8-19$ & I (0.68\%) & 57 (28.08\%) & \\
\hline $20-35$ & I $(0.68 \%)$ & $23(11.33 \%)$ & \\
\hline GAD-7 score & $3.08 \pm 1.90$ & $9.04 \pm 5.59$ & $<0.001$ \\
\hline $0-4$ & 105 (71.92\%) & $54(26.60 \%)$ & \\
\hline $5-21$ & $4 \mid(28.08 \%)$ & 149 (73.40\%) & \\
\hline PHQ-9 score & $3.82 \pm 2.17$ & $11.74 \pm 6.73$ & $<0.001$ \\
\hline $0-4$ & 86 (58.90\%) & $33(16.26 \%)$ & \\
\hline $5-27$ & $60(41.10 \%)$ & I 70 (83.74\%) & \\
\hline PSQI score & $4.88 \pm 3.21$ & $9.59 \pm 5.12$ & $<0.001$ \\
\hline $0-5$ & 76 (52.05\%) & $50(24.63 \%)$ & \\
\hline $6-10$ & 52 (35.62\%) & $58(28.57 \%)$ & \\
\hline $11-15$ & $8(5.48 \%)$ & 69 (33.99\%) & \\
\hline$|6-2|$ & $10(6.85 \%)$ & 26 (| $2.81 \%)$ & \\
\hline
\end{tabular}

Note: Values are presented as mean \pm standard deviation or number (\%).

Abbreviations: Acquired PE, acquired premature ejaculation; IELT, intravaginal ejaculatory latency time; PEDT, Premature Ejaculation Diagnostic Tool; IIEF-5, International Index of Erectile Function-5; IPSS, International Prostate Symptom Score; GAD-7, Generalized Anxiety Disorder-7; PHQ-9, The Patient Health Questionnaire-9; PSQI, Pittsburgh Sleep Quality Index.

\section{Study on the Risk Factors Associated with APE Patients}

We applied a binary classification logistic regression analysis to assess the impact of age, BMI, smoking history, erectile function, prostatitis-like symptoms, anxiety, depression and sleep quality on APE. PSQI score greater than 5 yielded a diagnostic sensitivity of $89.6 \%$ and specificity of $86.5 \%$ in distinguishing good and poor sleepers. ${ }^{22}$ According to the

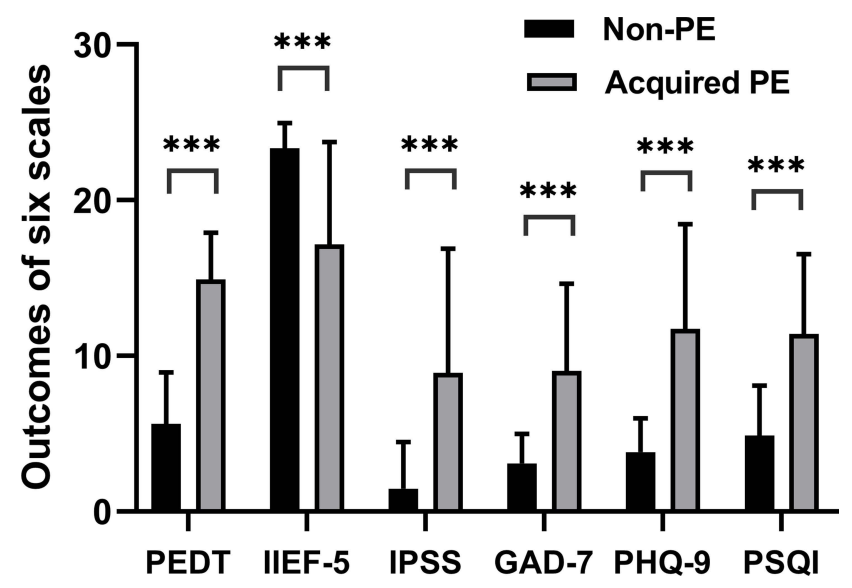

Figure I Outcomes of six scales in the APE and the non-PE subjects $(* * * \mathrm{P}<0.00 \mathrm{I})$. 

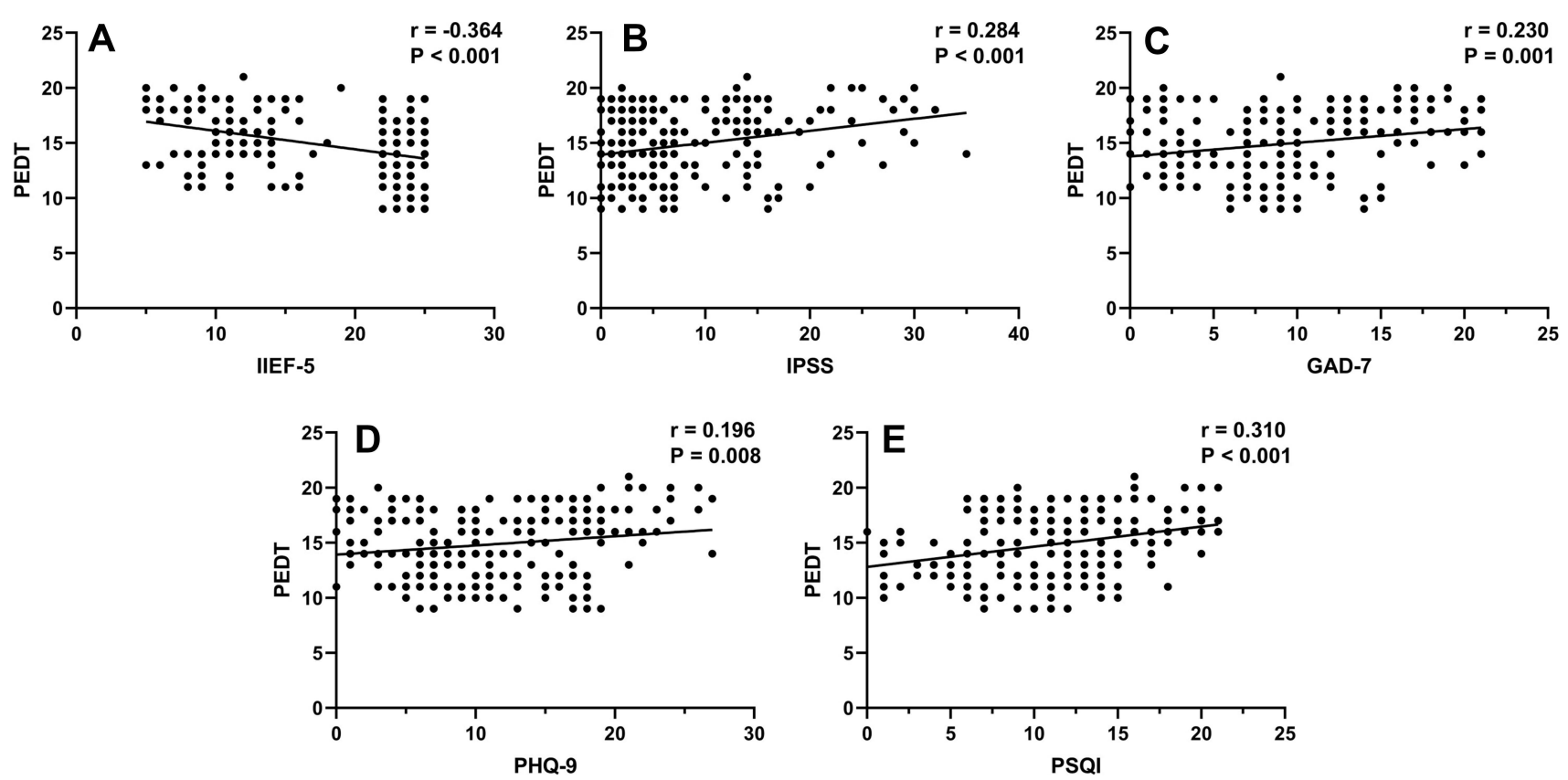

Figure 2 Correlation between clinical characteristics of patients and PEDT score. There was a significant negative correlation between PEDT and IIEF-5 (A) in APE group; there was a significant positive correlation between PEDT and IPSS (B) /GAD-7 (C) /PHQ-9 (D) /PSQI (E) in APE group.

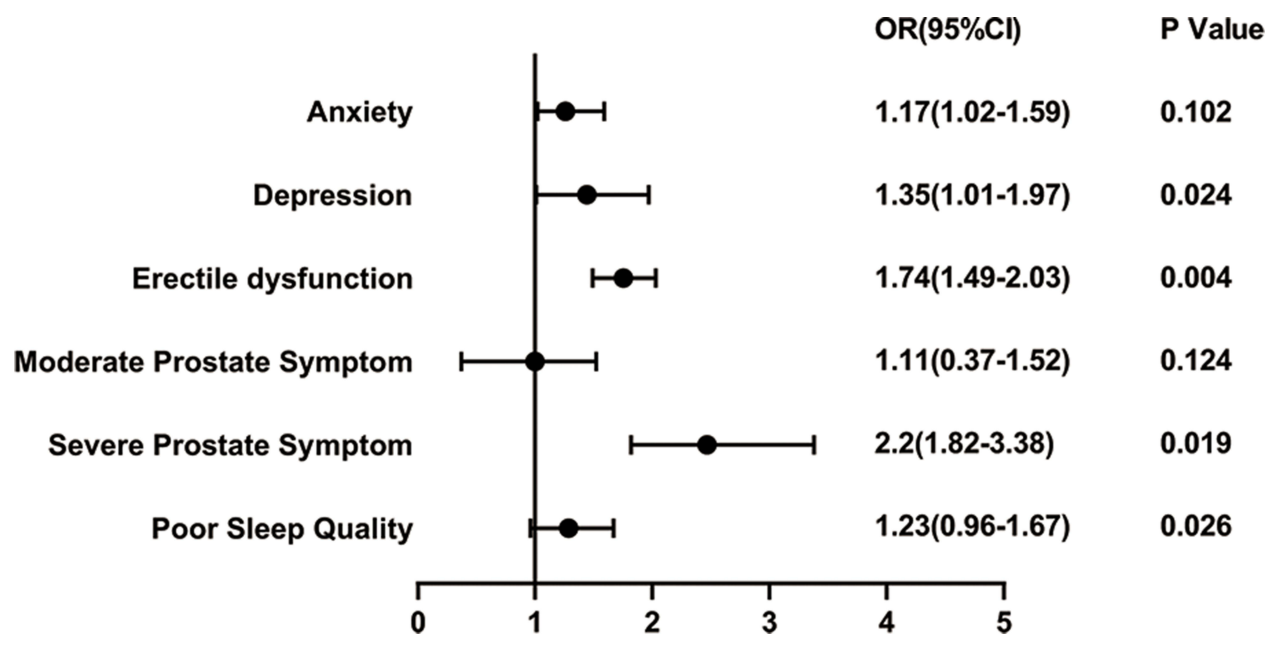

Figure 3 Logistic regression analysis of different risk factors in relation to acquired premature ejaculation.

above criteria, we divided the patients into two groups with different sleep quality. The results of the univariate analysis showed that erectile function, prostatitis-like symptoms, anxiety, depression, and sleep quality were identified as being significantly $(\mathrm{p}<0.05)$ associated with APE. After adjusting for the relevant factors, further multivariate analysis showed that erectile dysfunction (OR 1.74, 95\% CI, 1.49-2.03, P = 0.004), depression (OR 1.35, 95\% CI, 1.01-1.97, $\mathrm{p}=0.024$ ), severe prostatitis-like symptoms (severe prostatitis-like symptoms vs mild prostatitis-like symptoms, OR 2.2, 95\% CI, $1.82-3.38, \mathrm{p}=0.019$ ), and poor sleep quality (OR 1.23, 95\% CI, $0.96-1.67, \mathrm{p}=0.026$ ) were high risk factors of APE (see Figure 3).

\section{Stratification Analysis}

We divided patients with APE into two groups according to whether they suffered from sleep quality problems (Table 3). We observed that the APE patients with poor sleep quality was distinctly higher than patients without poor sleep quality 
Table 3 Prevalence of Anxiety, Depression, Erectile Dysfunction, Prostatitis-Like Symptoms Among APE Men with Different Sleep Quality

\begin{tabular}{|c|c|c|}
\hline \multirow[t]{2}{*}{ Variables } & \multicolumn{2}{|c|}{ Men with Acquired PE } \\
\hline & $\begin{array}{l}\text { With Poor Sleep Quality } \\
\qquad(n=153)\end{array}$ & $\begin{array}{l}\text { Without Poor Sleep Quality } \\
\qquad(n=50)\end{array}$ \\
\hline GAD-7 score & $10.56 \pm 5.46$ & $8.54 \pm 5.56$ \\
\hline Prevalence of Anxiety, n (\%) & $12 \mid(79.1 \%)$ & $28(56 \%)$ \\
\hline PHQ-9 score & $13.30 \pm 7.07^{*}$ & $11.24 \pm 6.56 *$ \\
\hline Prevalence of Depression, n (\%) & I 32 (86.3\%) & 38 (76\%) \\
\hline IIEF-5 score & $16.52 \pm 7.18$ & $17.37 \pm 6.37$ \\
\hline Prevalence of ED, n (\%) & $85(55.6 \%)$ & $22(44 \%)$ \\
\hline IPSS score & $1 \mathrm{I} .62 \pm 8.24 * *$ & $8.03 \pm 7.57 * *$ \\
\hline Prevalence of Mild PS, n (\%) & 91 (59.5\%) & $32(64 \%)$ \\
\hline Prevalence of Moderate PS, $n$ (\%) & 45 (29.4\%) & $12(24 \%)$ \\
\hline Prevalence of Severe PS, n (\%) & 17 (II.I\%) & $6(12 \%)$ \\
\hline
\end{tabular}

Notes: Values are presented as mean \pm standard deviation or number $(\%) . * 0.05, * *<0.01$.

Abbreviations: Acquired PE, acquired premature ejaculation; GAD-7, Generalized Anxiety Disorder-7; PHQ-9, The Patient Health Questionnaire-9; IIEF-5, International Index of Erectile Function-5; IPSS, International Prostate Symptom Score; PS, Prostatitis-Like Symptoms.

on the total prevalence of depression, the severity of prostatitis-like symptoms, and the average score of PHQ-9 and IPSS. There was no statistical significance on the total prevalence of anxiety, erectile dysfunction, and the score of GAD7 and IIEF-5 between the two groups. Furthermore, we divided the patients into four groups to show the distribution of GAD-7, PHQ-9, IPSS, and IIEF-5 scores of patients with different PSQI scores. The result is shown in Figure 4.

\section{Discussion}

Premature ejaculation (PE) is the most common male sexual dysfunction. While the exact etiology of PE has not been elucidated, relevant literature has reported several pathological conditions have been associated with PE. Although all these conditions should be considered as possible causes of APE, they can also worsen ejaculatory control in patients complaining of LPE. ${ }^{23}$ Previous studies have shown that lifelong premature ejaculation in humans may be explained by a hyposensitivity of the 5-HT2C and/or hypersensitivity of the 5-HT1A receptors, ${ }^{24}$ while acquired premature ejaculation is mainly affected by related secondary factors, such as chronic prostatitis, erectile difficulties and other comorbidities. ${ }^{25}$ A large demographic study found that PE patients were older and had higher BMI than the non-PE group, stratified analysis in the study showed that the APE group had severe anxiety and depression. ${ }^{26}$ Another study only included APE patients found that urinary tract symptoms, erectile dysfunction, hypogonadism and prostatitis, metabolic syndrome may be risk factors for acquired premature ejaculation. ${ }^{27}$ In addition, premature ejaculation may negatively impact the perception of orgasmic pleasure, both LPE and APE patients experienced a lower orgasmic intensity compared to the healthy controls. ${ }^{28}$

In 2018, Liu et $\mathrm{al}^{29}$ have concluded that psychological state and sleep quality might be closely related to PE. In contrast to the above study, our study only included patients with acquired premature ejaculation, because of acquired premature ejaculation is usually accompanied by comorbidities compared with patients with primary premature ejaculation. In this study, we explored the association between APE and various risk factors that may affect ejaculation function through multivariate and univariate analysis. After adjusting for the relevant factors, the results showed that erectile dysfunction, severe prostatitis-like symptoms, and poor sleep quality affected the presence of premature ejaculation. Our study also found that men with APE were older and had higher BMI than the non-PE group, and the APE group had lower IIEF-5 scores and higher IPSS/GAD-7/PHQ-9/PSQI scores compared with the non-PE group. Additionally, our study showed that PEDT was associated with IPSS/GAD-7/PHQ-9/PSQI scores positively and associated with IIEF-5 scores negatively. The stratified analysis of sleep quality showed that APE patients with different sleep quality have different prevalence rates of anxiety, depression, prostatitis-like symptoms, and erectile function, and there were 
PSQI and GAD-7

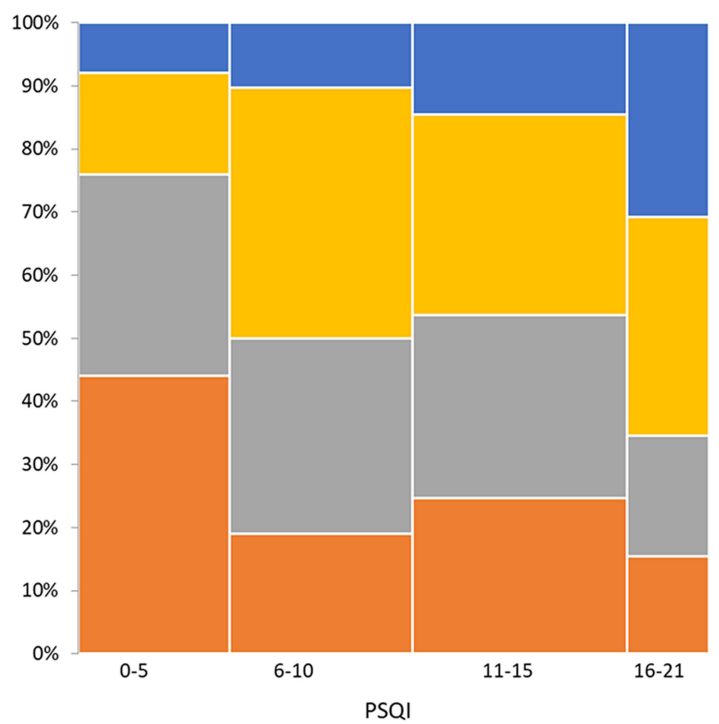

PSQI and IPSS

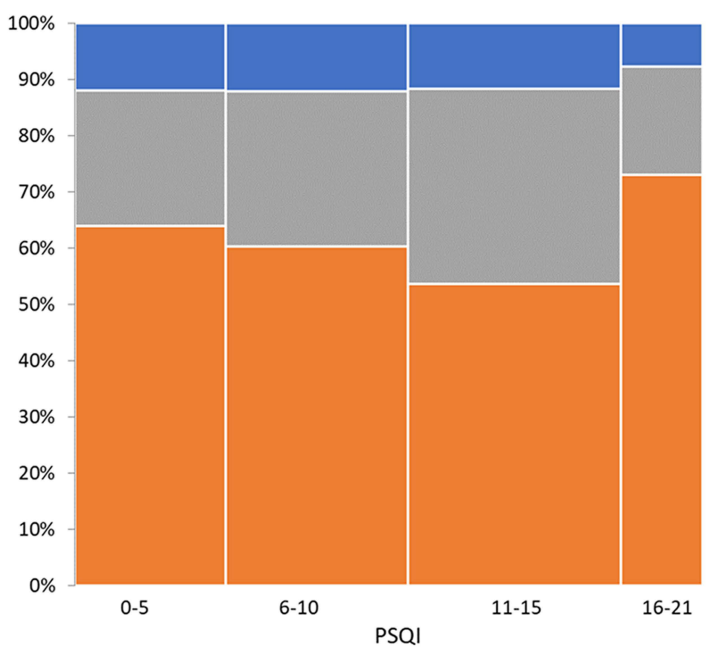

PSQI and PHQ-9

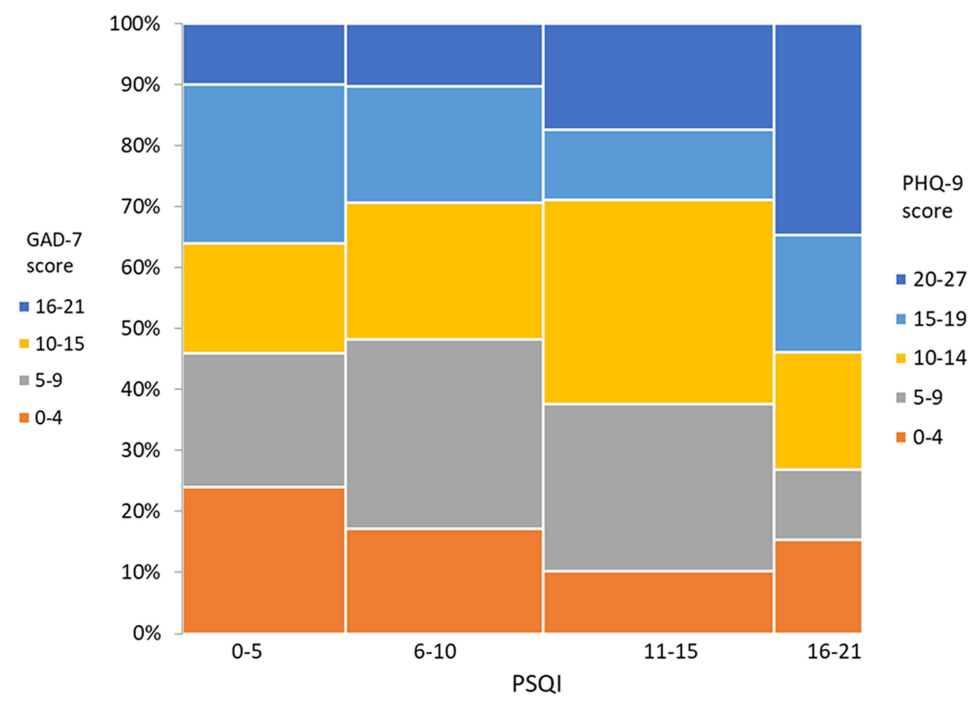

PSQI and IIEF-5

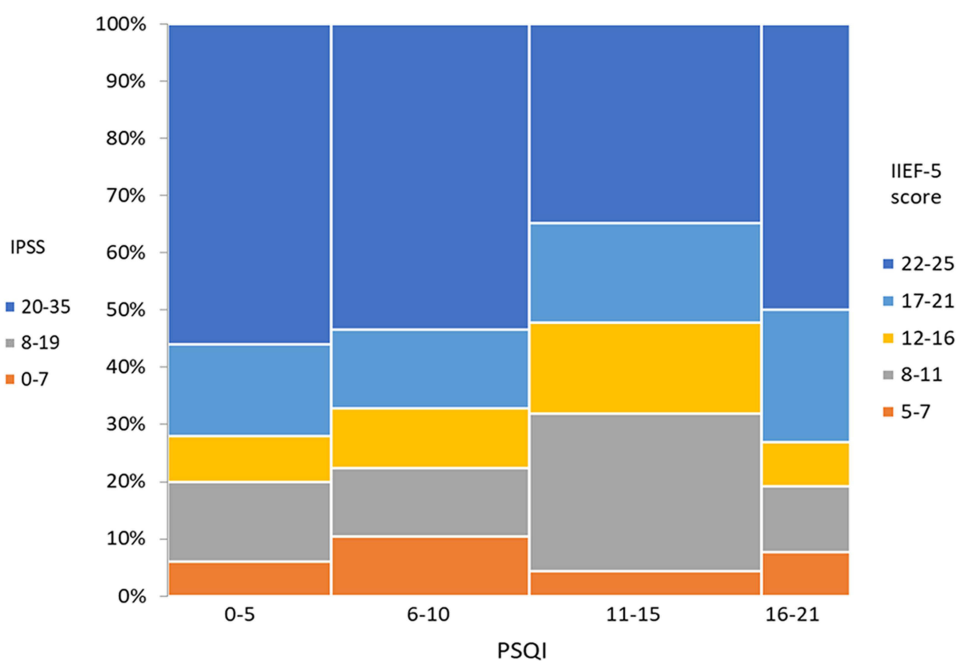

Figure 4 Comparison of clinical features of APE patients with different PSQI (all P< 0.05).

significant differences in GAD-7/PHQ-9/IPSS and IIEF-5 scores. Because of the findings mentioned above, we proposed that poor sleep quality may be one of the risk factors for acquired premature ejaculation.

Sleep that is insufficient, misaligned, or disrupted could impair the biological processes of males and lead to hypogonadism, erectile dysfunction, and infertility. There may also be a link between sleep and ejaculation. Serotonin and its central system receptors have been shown to play a pivotal role in both ejaculations and regulating sleepawakening and emotional state. Intervention on sleep state will also affect the expression of 5-HT and its relevant receptors. Related animal experiments have shown that sleep deprivation in rats can affect the expression of central 5-HT receptors and further lead to depressive behavior. Studies have also confirmed that sleep deprivation can interfere with rats' sexual behavior, and the frequency of ejaculation in rats will change after sleep deprivation.

Our research has shown that poor sleep quality could be a risk factor for acquired premature ejaculation because of a long-term sleep intervention may affect central ejaculation regulation. Besides, patients with poor sleep quality were more likely to suffer from erectile dysfunction and depression, while previous studies have confirmed that erectile dysfunction and depression can affect ejaculation function. ${ }^{30,31}$ Therefore, this could be one of the causes of ejaculation dysfunction in patients with sleep disorders. 
The present study has some limitations. We diagnose PE through the self-reported ejaculation latency questionnaire, which may be biased. Most of the clinical characteristics of patients, such as sleep quality, are also based on self-reports, which is not as objective as polysomnography and may lead to deviations in the results. In addition, our research is a cross-sectional survey, and further research is needed to explore the mechanism of sleep affecting ejaculation. Despite these limitations, our research is still meaningful. We comprehensively analyzed the possible risk factors for APE and confirmed that sleep quality is related to APE. We implemented the stratified analysis method to evaluate the impact of different sleep quality of APE patients on different complications such as ED and depression.

\section{Conclusion}

In general, sleep quality may be a potential risk factor for patients with acquired premature ejaculation. Our research revealed the impact of sleep quality on premature ejaculation and provided new viewpoints for further understanding and perfecting the pathogenesis of premature ejaculation.

\section{Abbreviations}

APE, acquired premature ejaculation; PEDT, Premature Ejaculation Diagnostic Tool; IIEF-5, International Index of Erectile Function-5; IPSS, International Prostate Symptom Score; GAD-7, Generalized Anxiety Disorder-7; PHQ-9, The Patient Health Questionnaire-9; PSQI, Pittsburgh Sleep Quality Index.

\section{Author Contributions}

All authors made a significant contribution to the work reported, whether that is in the conception, study design, execution, acquisition of data, analysis and interpretation, or in all these areas; took part in drafting, revising or critically reviewing the article; gave final approval of the version to be published; have agreed on the journal to which the article has been submitted; and agree to be accountable for all aspects of the work. All authors read and approved the submitted version.

\section{Funding}

This work received funding from the National Natural Science Foundation of China (Grant No. 8207061072).

\section{Disclosure}

The authors report no conflicts of interest.

\section{References}

1. Lee SW, Lee JH, Sung HH, et al. The prevalence of premature ejaculation and its clinical characteristics in Korean men according to different definitions. Int J Impot Res. 2013;25(1):12-17. doi:10.1038/ijir.2012.27

2. Serefoglu EC, Direk N, Hellstrom WJ. Premature ejaculation and erectile dysfunction prevalence and attitudes in the Asia-pacific region-a comment. J Sex Med. 2012;9(5):1488-1489. doi:10.1111/j.1743-6109.2012.02685.x

3. Schapiro B. Premature ejaculation, a review of 1130 cases. J Urol. 1943;50(3):374-379. doi:10.1016/S0022-5347(17)70462-4

4. Serefoglu EC, McMahon CG, Waldinger MD, et al. An evidence-based unified definition of lifelong and acquired premature ejaculation: report of the second international society for sexual medicine ad hoc committee for the definition of premature ejaculation. Sex Med. 2014;2(2):41-59. doi:10.1002/sm2.27

5. Colonnello E, Ciocca G, Limoncin E, Sansone A, Jannini EA. Redefining a sexual medicine paradigm: subclinical premature ejaculation as a new taxonomic entity. Nat Rev Urol. 2021;18(2):115-127. doi:10.1038/s41585-020-00417-1

6. Jannini EA, Ciocca G, Limoncin E, et al. Premature ejaculation: old story, new insights. Fertil Steril. 2015;104(5):1061-1073. doi:10.1016/j. fertnstert.2015.08.035

7. Institute of Medicine Committee on Sleep M, Research. The National Academies Collection: reports funded by National Institutes of Health. In: Colten HR, Altevogt BM, editors. Sleep Disorders and Sleep Deprivation: An Unmet Public Health Problem. Washington (DC): National Academies Press (US) Copyright (C) 2006, National Academy of Sciences; 2006.

8. Wise LA, Rothman KJ, Wesselink AK, et al. Male sleep duration and fecundability in a North American preconception cohort study. Fertil Steril. 2018;109(3):453-459. doi:10.1016/j.fertnstert.2017.11.037

9. Liu PY, Caterson ID, Grunstein RR, Handelsman DJ. Androgens, obesity, and sleep-disordered breathing in men. Endocrinol Metab Clin North Am. 2007;36(2):349-363. doi:10.1016/j.ecl.2007.03.002

10. Hoyos CM, Melehan KL, Phillips CL, Grunstein RR, Liu PY. To ED or not to ED-is erectile dysfunction in obstructive sleep apnea related to endothelial dysfunction?. Sleep Med Rev. 2015;20:5-14. doi:10.1016/j.smrv.2014.03.004 
11. Donatucci CF. Etiology of ejaculation and pathophysiology of premature ejaculation. J Sex Med. 2006;3(Suppl 4):303-308. doi:10.1111/j.17436109.2006.00305.x

12. Waldinger MD, Berendsen HH, Blok BF, Olivier B, Holstege G. Premature ejaculation and serotonergic antidepressants-induced delayed ejaculation: the involvement of the serotonergic system. Behav Brain Res. 1998;92(2):111-118. doi:10.1016/S0166-4328(97)00183-6

13. Monti JM. Serotonin control of sleep-wake behavior. Sleep Med Rev. 2011;15(4):269-281. doi:10.1016/j.smrv.2010.11.003

14. Azizi H, Hwang J, Suen V, et al. Sleep deprivation induces changes in 5-HT actions and 5-HT(1A) receptor expression in the rat hippocampus. Neurosci Lett. 2017;655:151-155. doi:10.1016/j.neulet.2017.06.053

15. Alvarenga TA, Hirotsu C, Mazaro-Costa R, Tufik S, Andersen ML. Impairment of male reproductive function after sleep deprivation. Fertil Steril. 2015;103(5):1355-1362.e1351. doi:10.1016/j.fertnstert.2015.02.002

16. Rosen RC, Riley A, Wagner G, Osterloh IH, Kirkpatrick J, Mishra A. The international index of erectile function (IIEF): a multidimensional scale for assessment of erectile dysfunction. Urology. 1997;49(6):822-830. doi:10.1016/S0090-4295(97)00238-0

17. Huang YP, Chen B, Ping P, et al. The premature ejaculation diagnostic tool (PEDT): linguistic validity of the Chinese version. J Sex Med. 2014;11 (9):2232-2238. doi:10.1111/jsm.12612

18. Barry MJ, Fowler FJ Jr., O’Leary MP, et al. The American Urological Association symptom index for benign prostatic hyperplasia. The Measurement Committee of the American Urological Association. J Urol. 1992;148(5):1549-1557; discussion 1564. doi:10.1016/S0022-5347 (17)36966-5

19. Löwe B, Decker O, Müller S, et al. Validation and standardization of the Generalized Anxiety Disorder Screener (GAD-7) in the general population. Med Care. 2008;46(3):266-274. doi:10.1097/MLR.0b013e318160d093

20. Kroenke K, Spitzer RL, Williams JBW. The PHQ-9. J Gen Intern Med. 2001;16(9):606-613. doi:10.1046/j.1525-1497.2001.016009606.x

21. Buysse DJ, Reynolds CF 3rd, Monk TH, Berman SR, Kupfer DJ. The Pittsburgh Sleep Quality Index: a new instrument for psychiatric practice and research. Psychiatry Res. 1989;28(2):193-213. doi:10.1016/0165-1781(89)90047-4

22. Shahid A, Wilkinson K, Marcu S, Shapiro CM. Pittsburgh Sleep Quality Index (PSQI). New York, NY: Springer; 2011.

23. Sansone A, Aversa A, Corona G, et al. Management of premature ejaculation: a clinical guideline from the Italian Society of Andrology and Sexual Medicine (SIAMS). J Endocrinol Invest. 2021;44(5):1103-1118. doi:10.1007/s40618-020-01458-4

24. Waldinger MD. The neurobiological approach to premature ejaculation. J Urol. 2002;168(6):2359-2367. doi:10.1016/S0022-5347(05)64146-8

25. Althof SE, Abdo CH, Dean J, et al. International Society for Sexual Medicine's guidelines for the diagnosis and treatment of premature ejaculation. J Sex Med. 2010;7(9):2947-2969. doi:10.1111/j.1743-6109.2010.01975.x

26. Yang Y, Lu Y, Song Y, Chen H, Liu X. Correlations and stratification analysis between premature ejaculation and psychological disorders. Andrologia. 2019;51(8):e13315. doi:10.1111/and.13315

27. Jeh SU, Yoon S, Choi JH. Metabolic syndrome is an independent risk factor for acquired premature ejaculation. World J Mens Health. 2019;37 (2):226-233. doi:10.5534/wjmh.180062

28. Limoncin E, Lotti F, Rossi M, et al. The impact of premature ejaculation on the subjective perception of orgasmic intensity: validation and standardisation of the 'Orgasmometer'. Andrology. 2016;4(5):921-926. doi:10.1111/andr.12220

29. Liu T, Jia C, Peng YF, Zhong W, Fang X. Correlation between premature ejaculation and psychological disorders in 270 Chinese outpatients. Psychiatry Res. 2019;272:69-72. doi:10.1016/j.psychres.2018.12.038

30. Kalejaiye O, Raheem AA, Moubasher A. Sleep disorders in patients with erectile dysfunction. BJU Int. 2017;120(6):855-860. doi:10.1111/ bju. 13961

31. Huang Y, Zhu M. Increased global PSQI score is associated with depressive symptoms in an adult population from the United States. Nat Sci Sleep. 2020;12:487-495. doi:10.2147/NSS.S256625

\section{Publish your work in this journal}

Nature and Science of Sleep is an international, peer-reviewed, open access journal covering all aspects of sleep science and sleep medicine, including the neurophysiology and functions of sleep, the genetics of sleep, sleep and society, biological rhythms, dreaming, sleep disorders and therapy, and strategies to optimize healthy sleep. The manuscript management system is completely online and includes a very quick and fair peer-review system, which is all easy to use. Visit http://www.dovepress.com/testimonials.php to read real quotes from published authors. 